\title{
Lower dose and shorter acquisition: Pushing the boundaries of myocardial perfusion SPECT
}

\author{
Oliver Gaemperli, MD, ${ }^{\mathrm{a}}$ and Philipp A. Kaufmann, $M D^{\mathrm{b}, \mathrm{c}}$
}

\section{See related article, pp. 847-857}

For the past three decades myocardial perfusion imaging SPECT (MPI-SPECT) has managed to prevail as one of the most important non-invasive imaging techniques in cardiology. The strengths of MPI-SPECT include the reliance on physiology rather than anatomical features of the coronary circulation, its standardized and efficient methodology, and a large body of evidence supporting its clinical value. However, while taking advantage of all these benefits inherent to the method, we have for long time neglected the need to push on technical developments and innovations and it may seem as if MPI-SPECT has missed the step into the twenty-first century. In fact, almost all around the globe, MPI-SPECT is still performed in a similar manner to 20 or 30 years ago using essentially the same radionuclides, protocols, and camera designs. In an era of emerging technologies and multimodality imaging, MP-SPECT has to compete with other techniques that are faster or avoid ionizing radiation such as CT coronary angiography or cardiac magnetic resonance. Thus, a technique which requires 20 - to 30-minute acquisition time and exposes the patient to $10-25 \mathrm{mSv}$ of ionizing radiation appears to be an endangered species in the cardiac imaging field.

However, recent years have witnessed important developments in device technology and a number of novel camera designs for dedicated cardiac MPI-SPECT have entered commercial production. ${ }^{1}$ Solid-state semiconductor detectors made of a cadmium-zinc-telluride (CZT) alloy provide direct conversion of absorbed

From the Cardiovascular Center, ${ }^{\text {a }}$ Department of Radiology, Cardiac Imaging, ${ }^{\text {b }}$ University Hospital Zurich, Zurich, Switzerland; and Zurich Center of Integrative Physiology (ZIHP), ${ }^{\mathrm{c}}$ University of Zurich, Zurich, Switzerland.

Reprint requests: Philipp A. Kaufmann, MD, Department of Radiology, Cardiac Imaging, University Hospital Zurich, NUK C 32, Raemistrasse 100, 8091 Zurich, Switzerland; pak@usz.ch.

J Nucl Cardiol 2011;18:830-2.

$1071-3581 / \$ 34.00$

Copyright (c) 2011 American Society of Nuclear Cardiology.

doi:10.1007/s12350-011-9410-z gamma-rays into an electrical signal and avoid the need for the bulky photomultiplier tubes used in conventional SPECT cameras. In 2008, GE Healthcare has introduced a CZT-based dedicated cardiac scanner (Discovery NM 530c) to the market. This device consists of 19 fixed arrays of pixilated CZT detectors assembled into a unique geometry that allows obtaining simultaneous views of the heart from different angles. This design coupled with the use of a proprietary multi-pinhole collimator improves count sensitivity compared to conventional devices while the CZT detector technology provides higher energy and spatial resolution. Indeed phantom studies have demonstrated improvements of 57-fold in count sensitivity, 1.7-2.5-fold in spatial resolution, and 1.65-fold in energy resolution. Similar technology has been introduced by other vendors. ${ }^{2}$

In nuclear cardiology image quality is determined to a large extent by high count statistics, which are based on a trade-off between injected radiotracer activity and duration of image acquisition. Thus, it can be anticipated that protocols consisting of lower radionuclide doses and shorter acquisition times would push the boundaries of image quality in conventional scanners. With higher sensitivity SPECT devices such as the Discovery NM $530 \mathrm{c}$, incentives to reduce acquisition times rather than radiation exposure to the patient were higher given the prospect for improved efficiency and patient throughput. Hence, a number of reports have been published demonstrating high image quality with CZT devices and comparable diagnostic performance with acquisition times as short as 2 minutes. ${ }^{3,4}$ However, the potential risks and hazards from ionizing radiation have obtained increasing public attention given to exponential growth of radiation exposure from medical imaging in the past years. And the recent devastating nuclear accidents with emission of unknown amounts of radionuclides into the environment have further sensitized the public to this issue. Therefore, nuclear cardiologists have been urged to reduce patients' radiation exposure from MPISPECT, if ever possible without increasing acquisition times and thereby reducing patient comfort. Some diagnostic centers have implemented stress-only MPSPECT protocols, thereby avoiding unnecessary rest studies in the presence of normal stress studies, which may reduce radiation exposure in a subgroup of patients. However, this approach requires an optimal set-up with 
nuclear physicians interpreting images immediately after scanning, and may pose logistic problems in centers that perform central reading at the end of the day. Positron emission tomography with either radiolabeled ${ }^{15} \mathrm{O}$-water or ${ }^{13} \mathrm{~N}$-ammonia is an excellent alternative to MPI-SPECT with a lower radiation exposure. However, availability is poor as these tracers are cyclotrondependent and their short physical half-lives render dissemination and use in PET centers without on-site cyclotron impossible.

In the present issue of the Journal of Nuclear Cardiology, Duvall et $\mathrm{al}^{5}$ make an important step forward in low-dose MPI-SPECT. In 131 consecutive patients, they assessed the feasibility of MPI with a reduced isotope dose (5-mCi rest and $15-\mathrm{mCi}$ stress) and imaging time (5-minute rest and 3-minute stress) compared to a protocol with the same doses but longer acquisition times ( 8 and 5 minutes) on a Discovery NM 530c CZT scanner. A subgroup of 27 patients underwent stress imaging on a conventional dual-head SPECT camera. There were no significant differences in image quality, summed perfusion scores, and inter- or intraobserver variability between short and long acquisition times. Similarly, when stress images were compared between CZT SPECT and conventional SPECT, total perfusion deficits were comparable while image quality was even superior with CZT SPECT. The effective dose for a whole study was $5.8 \mathrm{mSv}$ which is half the dose from a conventional full-dose one day rest/stress protocol with ${ }^{99 \mathrm{~m}} \mathrm{Tc}$-tracers.

The ability of the CZT device to obtain high image quality at low doses is explained by its high count sensitivity based on the above-mentioned geometric alignment of CZT detectors, which allows simultaneous acquisition of all required views. The potential loss of image resolution with multipinhole collimation compared to parallel-hole collimation is compensated by the higher energy resolution of the CZT detector (in the range of $5.7 \%$ compared to $9.4 \%$ with sodium iodide detectors) which can reduce the scatter component of total counts by $30 \%$. Indeed, in the present report by Duvall and colleagues, total count rates were double as high with the CZT device compared to conventional SPECT. Although this underlines the higher sensitivity of the CZT device, the numbers are lower than reported from phantom studies. A previous study documented a 7.22-fold increase in count rate sensitivity for a point source, a 6.34-fold, increase using a cardiac phantom, and a 4.1-7.0-fold increase for clinical perfusion studies. ${ }^{1}$ However, the comparison of the small field-of-view CZT camera with a conventional dual-head camera should be interpreted with caution as count rates are likely to be overestimated by the large field-of-view device due to greater contribution from extracardiac activity. This is further supported by the slightly higher image quality in the CZT images reported by Duvall and colleagues. Additionally, left ventricular ejection fraction and volumes from the CZT device correlated well with conventional gated SPECT. This represents further evidence of a comparable performance of the low-dose short acquisition time protocol as gated SPECT is particularly prone to artefacts with low count statistics.

The ability to reduce the effective dose of a full rest/ stress gated MPI-SPECT study represents a major step forward in nuclear cardiology and is crucial to maintain MPI-SPECT competitive in the cardiac imaging arena. This study provides the first proof that the concept of reducing injected dose while at the same time shortening image acquisition protocols in MP-SPECT is feasible and reproducible and provides high-quality images. However, it should be noted that the present manuscript consisted of a comparison with a reduced-dose protocol with longer acquisition times ( 8 and 5 minutes, respectively) whereas only a subgroup was compared to the established conventional method. Therefore, further studies in larger cohorts comparing image quality and perfusion information to conventional dual-head scanners and direct comparisons to the gold standard invasive coronary angiography are needed to consolidate the clinical usefulness of this novel approach. The reduction of radiation exposure and duration of image acquisition is a long-awaited step forward in nuclear cardiology, and it seems that the technology to reach this goal is finally in our hands. Thanks to these achievements, nuclear cardiology will remain to play a vital role in the assessment of patients with coronary artery disease alongside with newer methods such as CT and cardiac magnetic resonance. Additionally, with lower radiation burden, MPI-SPECT will become a more attractive modality for incorporation into multimodality or hybrid imaging systems. ${ }^{6}$ With these perspectives at hand, it truly appears as if conventional nuclear cardiology has finally taken the step into the new century and embarked into a new era of MPI-SPECT.

\section{References}

1. Slomka PJ, Patton JA, Berman DS, Germano G. Advances in technical aspects of myocardial perfusion SPECT imaging. J Nucl Cardiol 2009;16:255-76.

2. Sharir T, Slomka PJ, Berman DS. Solid-state SPECT technology: Fast and furious. J Nucl Cardiol 2010;17:890-6.

3. Buechel RR, Herzog BA, Husmann L, Burger IA, Pazhenkottil AP, Treyer V, et al. Ultrafast nuclear myocardial perfusion imaging on a new gamma camera with semiconductor detector technique: First clinical validation. Eur J Nucl Med Mol Imaging 2010;37:773-8.

4. Herzog BA, Buechel RR, Katz R, Brueckner M, Husmann L, Burger IA, et al. Nuclear myocardial perfusion imaging with a 
cadmium-zinc-telluride detector technique: Optimized protocol for scan time reduction. J Nucl Med 2010;51:46-51.

5. Duvall WL, Croft LB, Ginsberg ES, Einstein AJ, Guma KA, George $\mathrm{T}$, et al. Reduced isotope dose and imaging time with a high-efficiency CZT SPECT camera. J Nucl Cardiol 2011. doi: 10.1007/s12350-011-9379-7.
6. Herzog BA, Husmann L, Landmesser U, Kaufmann PA. Low-dose computed tomography coronary angiography and myocardial perfusion imaging: Cardiac hybrid imaging below $3 \mathrm{mSv}$. Eur Heart J 2009;30:644. 